\title{
A new variable step size block backward differentiation formula for solving stiff initial value problems
}

\begin{abstract}
A new block backward differentiation formula of order 4 with variable step size is formulated. By varying a parameter in the formula, different sets of formulae with A-stability property can be generated. At the cost of an additional function evaluation, the accuracy of the method is seen to outperform some existing backward differentiation formula algorithms. The strategy involved in controlling the step size ratio is also described. The problems tested with the method show its efficiency in solving stiff initial value problems.
\end{abstract}

Keyword: 2-point block method; Block backward differentiation formula; Initial value problems; Stiff; Variable step size 